\title{
15 years of Nature Chemical Biology
}

\author{
Over the past decade and a half, chemical biology has crystallized as a discipline and extended its reach into new \\ scientific areas, but the field's greatest promise lies ahead.
}

N ature Chemical Biology launched in June of 2005 with the primary aim of creating a "top-tier international journal that reflects the diversity and excitement of chemical biology research". Steered by the enthusiasm and engagement of our multidisciplinary community, our editorial team has remained committed to our original mission. We have witnessed the emergence of a global community who coalesced around the idea that science advances when researchers collaborate in asking insightful questions and developing creative and cross-disciplinary approaches to answer them. To this day, chemical biologists continue to support and inspire us at Nature Chemical Biology, and we remain humbled and grateful.

In the past 15 years, chemical biology's influence has expanded in both direct and subtle ways. Chemical biologists have made fundamental discoveries, such as uncovering previously unknown cell death pathways, conceptualizing the field of epitranscriptomics and defining frameworks for G-protein-coupled receptor signaling. Chemical biology has pushed mechanistic investigations toward greater molecular detail, contributing insights in areas like microbiome interactions, metal ion homeostasis, plant hormone action and riboswitch function. Further, the field's emphasis on methods development has stocked our scientific toolkit with chemical probes, analytical techniques, profiling reagents and optogenetic systems, as well as designed proteins, metabolic pathways and synthetic circuits, all of which have enriched our understanding of biology, expanded our capacity to engineer living systems and enabled pharmaceutical and biotechnological development.

In our 15th anniversary issue, we look back on the field's achievements over the past five years. First, we identified some noteworthy studies that have appeared in Nature Chemical Biology since our 10th anniversary and highlighted them in our "Greatest Hits" feature. In addition, following on the success of our "Voices of Chemical Biology" series (Nat. Chem. Biol. 11, 378-379; 2015), we asked an international group of chemical biologists to share their thoughts about recent trends in the field. We are pleased to present a cross section of the insightful answers that we received.
The "Voices" responses reflect the expansive scientific interests and enthusiasm of the chemical biology community. It is noteworthy that two topics-targeted protein degradation by degrons, PROTACs and 'molecular glues' and genome editing by CRISPR-Cas systems and base editorswere at the top of many scientists' lists of game-changing discoveries. Researchers also noted the influence of cryo-electron microscopy and mass spectrometry, while others highlighted single-cell approaches, artificial intelligence and machine learning as emerging contributors in chemical biology. Finally, many commented how in vivo chemical biology has taken shape, especially in the context of physiology and translational medicine.

In this special issue, we also include original research studies that provide a glimpse of today's chemical biology. The identification of chemical probes for a kinase linked to pancreatic cancer, for a phospholipase involved in lipid signaling and for cryptochromes involved in circadian rhythms highlights the enduring utility of small molecules for probing biology. Several research tools-methods for tRNA charging by ribozymes, detecting mammalian DNA methylation and sensing nitric oxide-underscore the persistence of technological innovation in the field. Lipidomic approaches reveal how membrane asymmetry affects protein localization, and mutational screening defines a membrane connection in $\alpha$-synuclein aggregation. Finally, mechanistic analysis of an enzymatic target of an antimicrobial agent and of synthetic gene circuits reinforce how molecular-level insights inform our understanding and manipulation of natural and designed systems.

In this issue, we also include two Perspective articles on foundational and emerging topics in chemical biology. The first Perspective, from Morrison, Podracky and Liu, provides a historical view of continuous directed evolution and highlights a thread of chemical biology in which molecular diversity coupled with selection techniques enables the evolution of biomolecules with new functions. A second Perspective from Diehl and Muir explores how chemical biology is revealing nascent regulatory connections between metabolism and chromatin modifications and defining the chemical and enzymatic reactions that mediate this small-moleculegenomic crosstalk.

Even as we celebrate chemical biology, we all currently find ourselves facing the grim realities and ongoing uncertainties created by the global COVID-19 pandemic. With most university classrooms, laboratories and companies temporarily closed, students and researchers have been forced to adapt. For many of us, these unusual circumstances have presented opportunities to reflect and, perhaps, reimagine our paths forward from here.

The field of chemical biology should do the same. Equipped with tools and the know-how to tackle complex interdisciplinary challenges, chemical biologists are already leading efforts to combat COVID-19: laboratories have retooled to support local diagnostic efforts, chemical libraries are being screened for potential antiviral treatments and global efforts to understand coronavirus biology have complemented the work of multidisciplinary teams racing to find safe and effective therapeutic antibodies and vaccines. To those chemical biologists contributing to these efforts, we collectively thank you!

More generally, chemical biologists are well positioned to enhance society's capacity to face global challenges. At this moment, the public's respect for science and technology is unparalleled, giving us a window of opportunity to advocate for robust science and technology policies, to demonstrate the scientific method as a model for data-driven decision making and to step forward as scientific and political leaders. Chemical biologists, who possess a breadth of knowledge and facility in communicating across disciplinary boundaries, should also emerge as leading educators in this teachable moment by advancing science education and literacy in their local communities and more broadly.

We look forward to working alongside chemical biologists, who are poised not only to expand interdisciplinary scientific discovery, but also to craft and implement a compelling new vision of science-based leadership in the years ahead.

Published online: 22 May 2020 https://doi.org/10.1038/s41589-020-0560-7 